Research Paper

\title{
Inhibition of RNA-Binding Protein Musashi-1 Suppresses Malignant Properties and Reverses Paclitaxel Resistance in Ovarian Carcinoma
}

\author{
Huaizeng Chen¹, Jia Liu², Hanzhi Wang1, Qi Cheng1, Caiyun Zhou³, Xiaojing Chen¹, Feng Ye1凶 \\ 1. Women's Reproductive Health Key Laboratory of Zhejiang Province, Women's Hospital, School of Medicine, Zhejiang University, Hangzhou, Zhejiang, \\ 310006, P.R. China; \\ 2. Department of Obstetrics and Gynecology, Women's Hospital, School of Medicine, Zhejiang University, Hangzhou, Zhejiang, 310006, P.R. China; \\ 3. Department of Pathology, Women's Hospital, School of Medicine, Zhejiang University, Hangzhou, Zhejiang, 310006, P.R. China
}

$\square$ Corresponding author: Feng Ye, MD \&PhD (yefyef@zju.edu.cn) Women’s Hospital, School of Medicine, Zhejiang University, Xueshi Rd \#1, Hangzhou, 310006, Zhejiang Province, P.R. China, Phone: 86-571-87061501, Fax: 86-571-87061878

(C) Ivyspring International Publisher. This is an open access article distributed under the terms of the Creative Commons Attribution (CC BY-NC) license (https://creativecommons.org/licenses/by-nc/4.0/). See http://ivyspring.com/terms for full terms and conditions.

Received: 2018.05.19; Accepted: 2019.01.12; Published: 2019.02.26

\begin{abstract}
Background and Aims: Ovarian carcinoma $(O C)$ is one of the most lethal malignant tumors with a high reoccurrence and chemoresistance. The key mechanism relationship with chemoresistance in ovarian carcinoma is still unclear. The existence of cancer stem cells involves in chemoresistance and reoccurrence in OC. The objective of this study was to investigate the expression, suppression of malignant properties and reversal of paclitaxel resistance inhibiting RNA-binding protein Musashi-1 with siRNA in ovarian cancer cells.

Methods: The expression of MSI-1 was analyzed in 39 normal ovarian epithelia tissues, 92 serous cystadenomas, 68 borderline serous cystadenomas, and 97 serous cystadenocarcinomas by immunohistochemistry. PLKO.1-MSI-1-siRNA expression vector was transfected into ovarian carcinoma cell line A2780 and its paclitaxel-resistant cell subline A2780/Taxol. The roles of MSI-1 in proliferation, apoptosis, migration and invasion were explored by cell proliferation analysis, Caspase 3 activity assay, wound healing assay, migration and matrigel invasion assay, respectively. Western Blotting and Real-time quantitative PCR were conducted to detect the expression of MSI- 1 and the ERK signaling pathway. Reversal of paclitaxel resistance assay was used to evaluate the role of MSI-1 in paclitaxel resistance of OC cells. Finally, therapeutic effects of MSI-1 inhibition were investigated the xenogratfs of SCID mice in vivo of the paclitacel-resistant.

Results: MSI- 1 is overexpressed and associated with an unfavorable prognosis in OC patients. Knockdown of MSI- 1 by small interfering RNA (siRNA) inhibits proliferation, promotes apoptosis, and reduces migration and invasion of cancer cells. Moreover, MSI-1 expression inhibition reverses paclitaxel-resistance in OC cells. We further display that MSI-1 effectively protects OC cells from paclitaxel-induced apoptosis by increasing the expression of $\mathrm{p}-\mathrm{Bcl}-2$ through ERK signaling pathway activation. In vivo, MSI-1 siRNA clearly showed a strong effect on tumor growth inhibition and paclitaxel-resistance reversal.

Conclusions: These findings suggest that MSI-1 overexpression is associated with the prognosis of OC patients, and knockdown of MSI- 1 can suppress malignant properties and reverse paclitaxel-resistance in OC cells. MSI-1 maybe act as a potential prognostic indicator and a therapeutic target in OC.
\end{abstract}

Key words: Musashi-1, PROC, siRNA, ERK1/2, Bcl-2

\section{Introduction}

Ovarian carcinoma (OC) is the second most common gynecological malignancy [1]. OC is usually diagnosed at an advanced stage, and despite cytoreductive surgery followed by combination chemotherapy, many ovarian cancer patients eventually experience reoccurrence with the development of chemoresistant tumors [2]. Based on extensive clinicopathologic and molecular genetic studies, a dualistic model of ovarian tumorigenesis has been proposed in which ovarian cancers can be divided into two subgroups: Type I, which are low-grade and histologically heterogeneous, and Type II, which are 
high-grade and mostly serous. Type II tumors, with the most common and lethal being high-grade serous ovarian carcinoma, represent $75 \%$ of all ovarian cancer and are responsible for $90 \%$ of ovarian cancer deaths [3]. Recently, a stem cell-like subtype of ovarian cancer has been reported [4]. This subtype causes poor disease-free as well as overall survival in patients with high-grade malignant ovarian cancer. This subtype is also associated with poorer diseasefree survival of patients with high-grade serous cancer, which has biological features consistent with Type II tumors [5].

Cancer stem cells (CSCs) are characterized by their enhanced ability of carcinogenesis and drug resistance. The potential of CSCs to enhance tumor formation and enable tumors to escape anti-tumor therapy is likely related with the expression of key regulatory gene [6]. One candidate protein is Musashi-1 (MSI-1). MSI-1 was first identified as an RNA-binding protein required for asymmetric division of sensory neuronal precursor cells [7]. It contains two N-terminal tandem RNA recognition motifs, each composed of two highly-conserved motifs (RNP-1 and RNP-2) [8,9]. Recently, MSI-1 expression has also been identified in a variety of solid tumors, including glioma, colorectal cancer and endometrial carcinoma [10-12]. In addition, Chen et al. reported that in a glioblastoma cell line, MSI-1 activated AKT and influenced AKT-derived IL-6 secretion in response to cisplatin (DDP), therefore contributing to chemoresistance and reoccurrence [13]. Therefore, MSI-1 is not only a marker of cancer stem cells but it also acts as a signaling molecule involved in protecting the cancer stem cells from the damages of chemo- or radio- treatment.

Our previous study, as well as other literature reports, showed that CSCs markers such as Nanog, Oct-4, SSEA4, Sox2, and Nestin are highly expressed in various ovarian cancer cell lines, patient ascites, and primary ovarian tumor specimens, and the increased expression of these CSCs markers is associated with poor prognosis and chemoresistance in ovarian cancer cells [14-19]. Up to now, the expression and role of MSI-1 in OC has not been investigated. Furthermore, the role of MSI-1 in metastasis and its association with the prognosis of ovarian carcinoma has not been well researched.

In the present study, we first detected the expression of MSI-1 in OC tissues and demonstrated a significant association between high MSI-1 expression and the clinicopathological characteristics of poor prognosis. Furthermore, we constructed a lentiviral vector expressing MSI-1 siRNA and transfected a paclitaxel-resistant OC cell line as well as the parental OC cell line. We found that silencing MSI-1 expression inhibited proliferation, promoted apoptosis, and reduced migration and invasion of cancer cells. Most importantly, we also found that MSI-1 induced ERK protein phosphorylation, thereby activating the anti-apoptotic protein Bcl-2 and reducing cancer cell apoptosis. Inhibition of MSI-1 reversed chemoresistance to paclitaxel in paclitaxel-resistant OC cell. Lastly, using a xenograft mouse model, we evaluated whether the decreased expression of MSI-1 has an effect on growth and resistance of cancer cells in vivo.

\section{Materials and Methods}

\section{Reagents and cell culture}

Paclitaxel (P106868) was purchased from Aladdin Industrial Corporation. Antibodies to MSI-1 (271851 -AP), $\beta$-actin (66009-1-Ig), ERK1/2(16443-1-AP), HRP-labeled secondary anti-mouse IgG $(\mathrm{H}+\mathrm{L})$ (SA000 01-1) and anti-rabbit IgG (H+L) (SA00001-2) antibodies were purchased from Proteintech, respectively. Antibodies to p-ERK 1/2 (sc-81492) and p-Bcl-2 (sc-37 7554), Lipofectamine 3000 Transfection Reagent (L300 0008) was purchased from Invitrogen. AllStars Negative Control siRNA(1027281) was purchased from QIAGEN. pLKO.1 puro plasmid was obtained from the nonprofit plasmid repository (Addgene). siRNA was synthesized by TaKaRa. Primers and DNA were synthesized by Sangon Biotech. CaspACE Assay System (colorimetric, G7351) was purchased from Promega. Transwell Permeable Supports (3428) was purchased from Corning. BD Matrigel ${ }^{\mathrm{TM}}$ Basement Membrane Matirx (356234) was purchased from BD.

Human lung fibroblasts Mrc-5, Human umbilical vein endothelial cell line ECV304, Human endometrial cancer cell line Ishikawa(IK), Human ovarian cancer cell line 3AO, Human ovarian cancer cell line A2780 and its paclitaxel-resistant cell subline A2780/ Taxol are preserved by Women's Reproductive Health Key Laboratory of Zhejiang Province. Mrc-5, ECV304, IK, 3AO, A2780 were maintained in RPMI 1640, supplemented with $10 \%$ fetal bovine serum (FBS) at $37^{\circ} \mathrm{C}$ and $5 \% \mathrm{CO}_{2}$. A2780/Taxol was cultured in RPMI-1640 containing 10\% FBS and 80nM paclitaxel.

\section{Patient selection and immunohistochemistry}

296 samples were obtained from the Pathological Department of the Women's Hospital at the Zhejiang University School of Medicine (China) between March 1997 and December 2008. The samples consisted of 39 normal ovarian epithelia tissues, 92 serous cystadenomas, 68 borderline serous cystadenomas, 97 serous cystadenocarcinomas (including 6 stage I, 28 stage II, 58 stage III and 5 stage IV). Patients who were treated with chemotherapy, immunotherapy or radiotherapy prior to surgery were excluded. All the patients underwent primary surgery followed 
by paclitaxel-based chemotherapy. For all patients, the following clinical parameters were collected: patient age; preoperative serum CA125; volume of ascites, tumor location (unilateral or bilateral), tumor maximum size, International Federation of Gynecology and Obstetrics (FIGO) stage; tumor differentiation grade, tumor residues after primary surgery, and chemotherapy sensitivity. Patients with progressive disease during primary chemotherapy or suffered recurrent disease within 6 months of completing primary chemotherapy were defined paclitaxel-resistant. Patients with recurrence beyond 6 months or without recurrence were defined paclitaxel-sensitive. The study was approved by the Ethics Committee of the Faculty of the Affiliated Women's Hospital, School of Medicine, Zhejiang University. All patients signed the informed consent.

$10 \%$ formalin was used to fix tissues. Antigen retrieval was performed by boiling the samples in 10 $\mathrm{mM}$ citrate buffer for $2 \mathrm{~min}$. Endogenous peroxidase activity was blocked in 3\% hydrogen peroxide for 10 min at room temperature (RT). Slides were incubated with MSI-1 antibodies (1:200) at RT for $1 \mathrm{~h}$ followed by incubation with Dako EnvisionTM Peroxidase (Dako Diagnostica, Hamburg, Germany) for $30 \mathrm{~min}$ at RT. 3,3'-diaminobenzidine tetrahydrochloride was applied for visualization, the slides were counterstained with hematoxylin. Negative controls were obtained by replacing the primary antibody with normal bovine serum.

The semiquantitative immunoreactive scoring system was evaluated based on the percentage of positive cells as well as the staining intensity (400x, ten random fields). Negative staining was defined as 0 , weak staining with faint yellow was 1 , moderate staining with brown yellow was 2 , and strong staining with dark yellow was 3 . For scoring the percentage of positive cells, $<5 \%$ was defines as $0,<25 \%$ was 1 , $<75 \%$ was 2 , and $>75 \%$ positive cells was defined as 3 . The symbolic designation was as follows: - (0), + (1-2), ++ (3-4), and +++ (5-6). The MSI-1 expression was assessed by two pathologists blinded to the clinicopathologic data. (As described in detail previously [20])

\section{siRNA interference}

We designed three pairs of siRNA according to MSI-1 gene sequence (NM_002442), using siDirect Ver2.0 (http://sidirect2.rnai.jp/) (Supplementary Table 1). All three pairs of siRNAs met the requirements of three algorithms simultaneously (Ui-Tei, Reynolds, and Amarzguioui). All of the siRNAs were chosen 100 nucleotides after the initiation codon, to avoid the $5^{\prime}$ and $3^{\prime}$ region of the UTR. The sense and the antisense strands have been tailed two bases at the $3^{\prime}$ terminal. Finally, three consecutive bases at the $5^{\prime}$ or $3^{\prime}$ end of the sense and antisense strands were 2'-OMe-4'-thioribonucleoside modified.

We used these three pairs of siRNAs (S1, S2, S3) to perform interference experiments, and the most effective pair of siRNAs was subsequently included in the lentiviral vector for functional experiments. The A2780 and A2780/Taxol, were transfected with $50 \mathrm{nM}$ S1, S2 and S3, and MSI-1 mRNA and protein were measured to identify the most effective siRNA. The dose radiant curve $(50 \mathrm{nM}, 10 \mathrm{nM}, 2 \mathrm{nM}, 400 \mathrm{pM}$, and $80 \mathrm{pM}$ ) was then performed with the selected siRNA.

A2780 and A2780/Taxol cells were seeded in a six-well plate at $0.5 \times 10^{6}$ cells per well. After $24 \mathrm{~h}$, the cells were transfected with siRNA using Lipofectamine 3000. The negative control was transfected with a non-specific control siRNA, while the blank group was transfected with water. $48 \mathrm{~h}$ and $72 \mathrm{~h}$ post transfection, mRNA and protein were measured.

Table 1. Association between MSI-1 expression and the clincial characteristics in epithelial ovarian serous cystadenocarcinoma.

\begin{tabular}{|c|c|c|c|c|c|c|c|}
\hline \multirow[t]{2}{*}{ Clinical characteristics } & \multirow[t]{2}{*}{$\mathbf{N}$} & \multicolumn{4}{|c|}{ MSI expression } & \multirow[t]{2}{*}{$\mathbf{r}$} & \multirow[t]{2}{*}{$\mathbf{P}$} \\
\hline & & $(-)$ & $(+)$ & $(++)$ & $(+++)$ & & \\
\hline \multicolumn{8}{|l|}{ Age } \\
\hline$\leq 45$ & 37 & 6 & 7 & 8 & 16 & 0.049 & 0.605 \\
\hline$>45$ & 60 & 7 & 11 & 14 & 28 & & \\
\hline \multicolumn{8}{|c|}{ Preoperative serum CA125 (U/ml) } \\
\hline$<500$ & 42 & 8 & 12 & 9 & 13 & $\underline{0.269}$ & $\underline{0.004}$ \\
\hline$\geq 500$ & 55 & 5 & 6 & 13 & 31 & & \\
\hline \multicolumn{8}{|l|}{ Volume of ascites $(\mathrm{ml})$} \\
\hline$<500$ & 62 & 8 & 12 & 14 & 28 & 0.002 & 0.981 \\
\hline$\geq 500$ & 35 & 5 & 6 & 8 & 16 & & \\
\hline \multicolumn{8}{|l|}{ Tunor side } \\
\hline Unilateral & 26 & 4 & 5 & 6 & 11 & 0.039 & 0.678 \\
\hline Bilateral & 71 & 9 & 13 & 16 & 33 & & \\
\hline \multicolumn{8}{|c|}{ Tumor maximum size $(\mathrm{cm})$} \\
\hline$\leq 10$ & 52 & 7 & 9 & 13 & 23 & 0.005 & 0.954 \\
\hline$>10$ & 45 & 6 & 9 & 9 & 21 & & \\
\hline \multicolumn{8}{|l|}{ FIGO stage } \\
\hline I & 6 & 4 & 1 & 1 & 0 & $\underline{0.352}$ & $\underline{0.000}$ \\
\hline II & 28 & 6 & 8 & 5 & 9 & & \\
\hline III & 58 & 3 & 8 & 15 & 32 & & \\
\hline IV & 5 & 0 & 1 & 1 & 3 & & \\
\hline \multicolumn{8}{|l|}{ Differentiation grade } \\
\hline I & 8 & 4 & 3 & 1 & 0 & $\underline{0.533}$ & $\underline{0.000}$ \\
\hline II & 35 & 5 & 12 & 12 & 6 & & \\
\hline III & 54 & 4 & 3 & 9 & 38 & & \\
\hline \multicolumn{8}{|l|}{ Optimal surgery } \\
\hline Yes & 73 & 11 & 15 & 19 & 28 & $\underline{0.202}$ & $\underline{0.032}$ \\
\hline No & 24 & 2 & 3 & 3 & 16 & & \\
\hline \multicolumn{8}{|c|}{ Chemotherapy sensitivity } \\
\hline Sensitive & 79 & 13 & 16 & 20 & 30 & $\underline{0.284}$ & $\underline{0.003}$ \\
\hline Resisitant & 18 & 0 & 2 & 2 & 14 & & \\
\hline
\end{tabular}

Underlined values show statistical data with significant difference. Kendall's tau-b correlation coefficients ( $r$ ) were applied by the Bivariate Correlation analysis. A P value less than 0.05 was considered significant.

\section{Cell transfection with pLKO.1-MSI-1-S2 expression vector}

The selected shRNA sequence was designed and 
cloned into the lentiviral eukaryotic expression vector pLKO.1-puro (Figure 1A). The S2 sequence was determined to be the most effective. The MSI-1-S2 sequence was as follows: forward, 5'-CCG GTG AAT TTC ACA CAC TTT CTC CAC TTC AAG AGA GTG GAG AAA GTG TGT GAA ATT CAT TTT TTG GTA CC-3', and reverse, 5'-AAT TGG TAC CAA AAA ATG AAT TTC ACA CAC TTT CTC CAC TCT CTT GAA GTG GAG AAA GTG TGT GAA ATT CA-3'. The shRNA fragment was cloned into the pLKO.1-puro vector using AgeI/EcoRI sites (Figure 1B). After KpnI digestion, the pLKO.1-MSI-1-S2 recombinant plasmid was obtained. $1 \times 10^{7} \mathrm{Mrc}-5$, ECV304, IK, 3AO, A2780 or A2780/Taxol cells were transfected with pLKO.1-MSI-1-S2 or pLKO.1-puro (negative control) using Lipofectamine 3000, and were selected with G418 (400 $\mathrm{\mu g} / \mathrm{ml})$ for 14 days.

\section{Real-time quantitative PCR (qPCR)}

Total RNA was treated with DNase I. cDNA was synthesized and used as a template for qPCR. The primers used were 5'-GTCTCGAGTCATGCCCTACG $-3^{\prime} ; 5^{\prime}$ - AGGAATGGCTGTAAGCTCGG -3'. $\beta$-actin was used as a loading control. All reactions were performed with a ViiA 7 Dx System (ABI). The $\Delta \mathrm{Ct}$ for gene-specific mRNA expression was calculated relative to the $\mathrm{Ct}$ of $\beta$-actin. Relative mRNA expression was calculated with the formula: $2^{-{ }^{-} \mathrm{CT}}$.

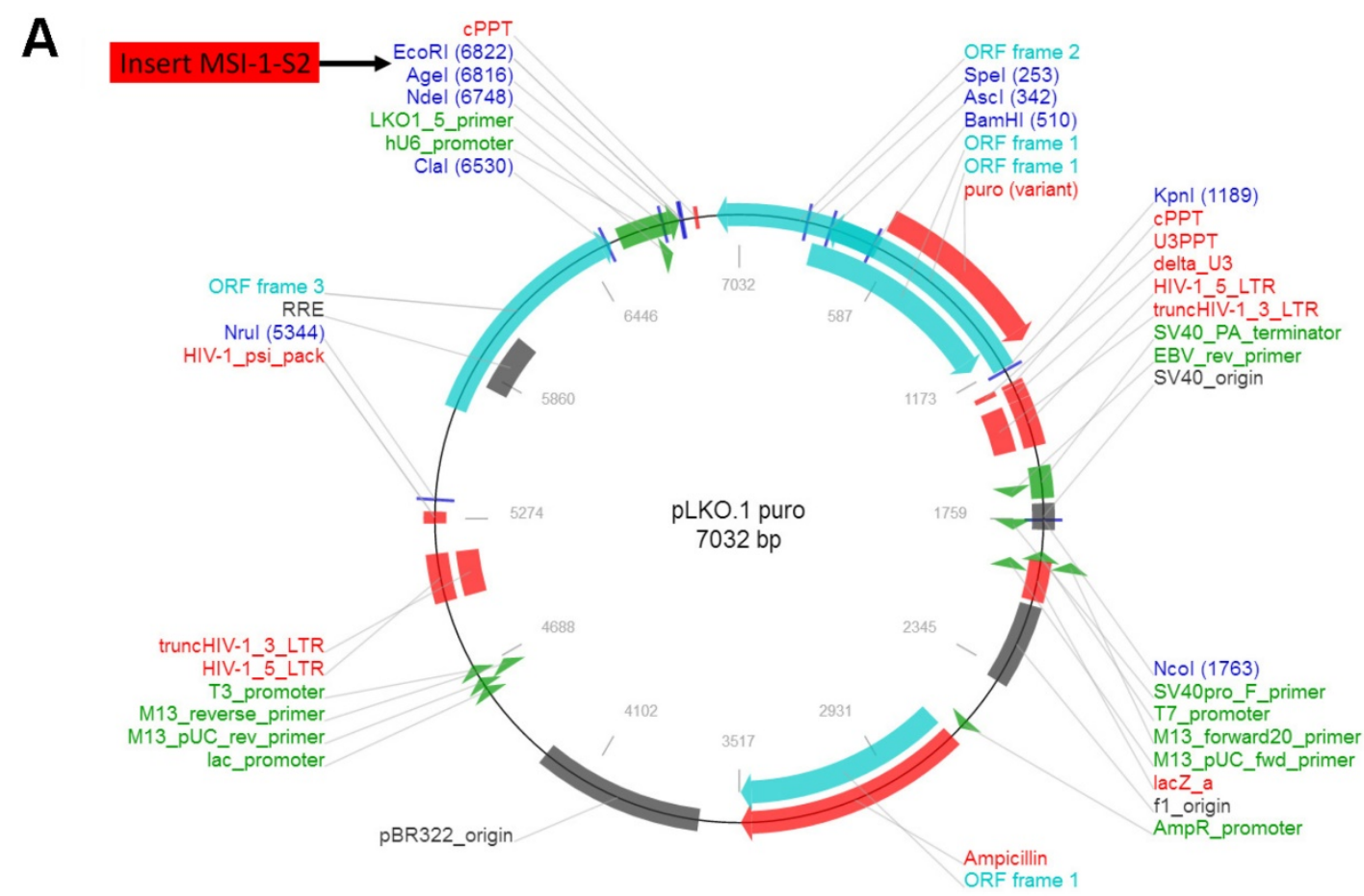

B

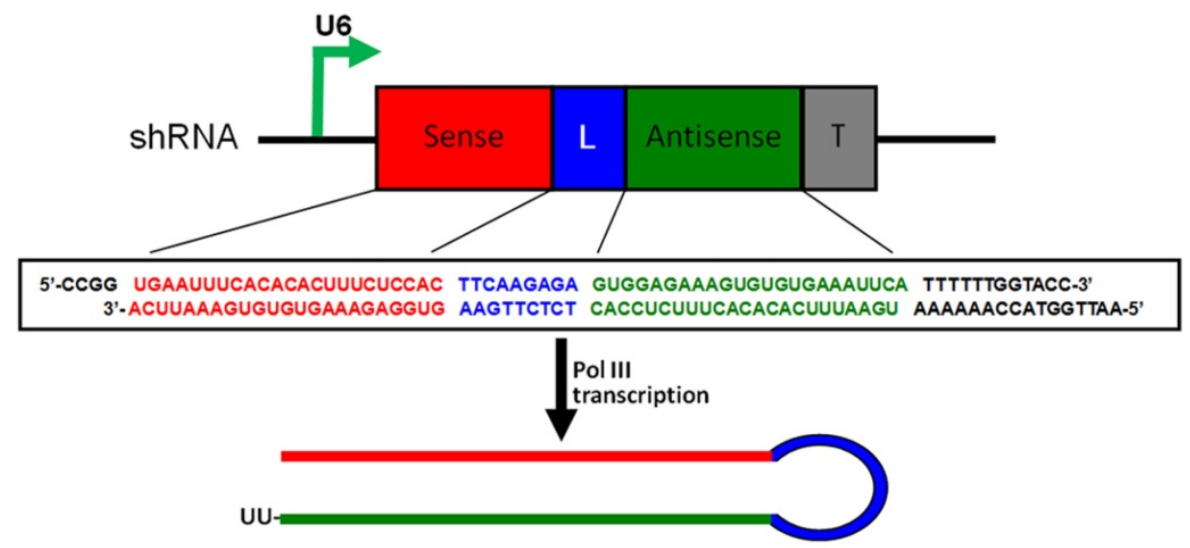

Figure 1. The construction of pLKO.1-MSI-1-S2 expression Vector. (A) Map of pLKO.1-MSI-1-S2 recombinant plasmid and its restriction site. (B) Small hairpin S2 schematic diagram. The U6 promoter guides transcription of small hairpin S2; includes 23 sense bases and 23 antisense bases of S2. 


\section{Western blotting}

After transfection, cells were lysed using RIPA lysis buffer. $10 \mu \mathrm{l}$ of each sample was loaded into an $8 \%$ polyacrylamide gel. Subsequently, proteins were transferred to a $0.45 \mu \mathrm{m}$ PVDF membrane. After blocking in 5\% non-fat milk for $1 \mathrm{~h}$, membranes were incubated with primary antibodies: MSI-1 (1:2000), ERK1/2 (1:1000), p-ERK 1/ 2 (1:500), p-Bcl-2 (1:500) or $\beta$-actin (1:5000) for $4 \mathrm{~h}$. Membranes were then washed with TBS containing $0.05 \%$ Tween- 20 followed by a $2 \mathrm{~h}$ incubation with an HRP-conjugated secondary antibody (1:5000). After a final wash, the membranes were imaged using an Image Quant LAS 4000 mini (GE Healthcare) with ECL.

\section{Cell proliferation analysis}

Cell proliferation was analyzed with the Cell Proliferation ELISA BrdU (colorimetric) kit. Absorbance (A) was measured at $370 \mathrm{~nm}$ (reference wavelength $492 \mathrm{~nm}$ ), and calculated using the formula: $\mathrm{A}_{\text {experiment }} / \mathrm{A}_{\text {control. }}$

\section{Caspase 3 activity detection}

After transfection, cells were collected and adjusted to $1 \times 10^{8}$ cells $/ \mathrm{ml}$. Cells were lysed for $15 \mathrm{~min}$ and spun at $15,000 \times g$ for $20 \mathrm{~min}$ to allow for collection of the supernatant. The activity of Caspase 3 was measured according to the CaspACE Assay System (colorimetric) manual. Absorbance was measured at $405 \mathrm{~nm}$.

\section{Wound healing assay}

A scratch was made using a $20 \mu \mathrm{l}$ pipette tip through confluent cells plated in six-well plates. After rinsing with PBS, cells were cultured in complete media. Photographs were taken at 0,24 and $48 \mathrm{~h}$ post wounding. All experiments were carried out in triplicate.

\section{Migration assay}

After transfection for $48 \mathrm{~h}$, migratory ability was tested using Transwell Permeable Supports with a pore size of $8 \mu \mathrm{m}$ (Corning). The upper chambers were loaded with $1 \times 10^{6}$ cells in $2 \mathrm{ml}$ of serum-free media. The lower chambers were filled with $2 \mathrm{ml}$ of media with $10 \%$ FBS. The chambers were incubated at $37^{\circ} \mathrm{C}$ and $5 \% \mathrm{CO}_{2}$ for $24 \mathrm{~h}$. The upper surface of the membranes were then gently scraped and washed with PBS to remove the stationary cells. The membranes were then fixed in $95 \%$ ethanol for $25 \mathrm{~min}$ followed by staining with hematoxylin. The number of migrated cells was counted and averaged between ten random fields per well.

\section{Matrigel invasion assay}

Matrigel stored at $-20^{\circ} \mathrm{C}$ was thawed at $4^{\circ} \mathrm{C}$, and then mixed with OPTI-MEM media (1:6) on ice. The upper surface of the membranes was coated with matrigel. The following steps were similar compared to the transwell migration assay.

\section{Reversal of Paclitaxel Resistance Assay}

In each group, $1 \times 10^{5}$ cells $/ \mathrm{ml}$ were resuspende, and cultured in 96 well plates for $24 \mathrm{~h}$. Paclitaxel was added to each group with $0,3.125,6.25,12.5,25,50$, 100 , and $200 \mu \mathrm{g} / \mathrm{ml}$ for $24 \mathrm{~h}$. The cell proliferation inhibition rate of paclitaxel was analyzed using the Cell Proliferation ELISA BrdU (colorimetric) kit. Absorbance (A) was measured at $370 \mathrm{~nm}$ (reference wavelength $492 \mathrm{~nm}$ ). The $\mathrm{IC}_{50}$ ratio of A2780/Taxol and its parent cell A2780 was considered the Resistant Folder (RF). The $\mathrm{IC}_{50}$ ratio of $\mathrm{A} 2780 /$ Taxol and A2780/Taxol transfected with pLKO.1-MSI-1-S2 was considered the Reversal Index (RI).

\section{Ovarian Cancer Xenogratfs in SCID mice}

Female CB-17 SCID mice (4-6 weeks old) and weighing 20-25g were purchased from Experimental Animal Center of Zhejiang Academy of Medical Science. SCID mice were maintained under specific pathogen-free conditions and cared for in accordance with all national and international standards. For the xenograft tumor growth assay, A2780/Taxol cells $\left(1 \times 10^{7}\right.$ cells/200ul in PBS with $50 \%$ Matrigel) were injected subcutaneously into the right flank of the SCID mice. Palpable solid tumors developed within 15 days post cell inoculation, and as soon as tumor volume reached $3-4 \mathrm{~mm}$ in diameter, the SCID mice were randomly divided into control group (treated with negative control siRNA) and treatment group (treated with MSI-1-S2). Three mice were in each group. Each tumor-bearing SCID mice received paclitacel at a dose $10 \mathrm{mg} / \mathrm{kg}$ by intravenous administration through the tail vein once per week for 4 consecutive weeks. The mice were treated with negative control siRNA $(1 \mathrm{mg} / \mathrm{kg})$ or MSI-1-siRNA2 $(1 \mathrm{mg} / \mathrm{kg})$ every 3 days for 5 weeks using intratumoral injection. After five weeks, SCID mice were sacrificed by cervical vertebra dislocation and necropsied. Tumor volumes were calculated using the formula $\mathrm{V}=$ $\left(\mathrm{L} \times \mathrm{W}^{2}\right) / 2, \mathrm{~L}$ is length and $\mathrm{W}$ is width [21].

\section{Statistical analysis}

The SPSS 18.0 software was used for statistical analyses. The Kruskal-Wallis $\mathrm{H}$ and the MannWhitney $U$ tests were used to evaluate MSI-1 expression. The Spearman and Kendall tests were applied to analyze the association between positive expression and clinicopathologic factors. All data is expressed as the mean $\pm S D$ and the statistical significance was calculated by ANOVA with a post hoc analysis (Fisher least significant difference) or a 
Student's two-tailed t-test as appropriate. The result was considered statistically significant when the $\mathrm{P}$ value was less than 0.05 , bilaterally.

\section{Results}

\section{High expression of MSI-1 protein is associated with clinicopathological characteristics of serous ovarian carcinoma}

Positive MSI-1 staining was mainly localized in the cytoplasm (Figure 2A). The expression intensity of MSI-1 in normal epithelium, serous cystadenoma, borderline serous cystadenoma, and serous cystadenocarcinoma showed a gradual rising trend.

Significant differences were observed among normal epithelium, serous cystadenoma, borderline serous cystadenoma, and serous cystadenocarcinoma $\left(X^{2}=74.644, P<0.001\right)$ (Figure 2B). With the exception of no statistical significance between normal tissue and serous cystadenoma, the pairwise comparisons of the remaining groups resulted in significant differences.

Stratified analysis displayed that there was no correlation between age, volume of ascites, tumor side (unilateral or bilateral), tumor maximum size and MSI-1 expression in serous cystadenocarcinoma. However, preoperative CA125 levels $(\mathrm{r}=0.269, \mathrm{P}=$ $0.004)$, FIGO stage $(\mathrm{r}=0.352, \mathrm{P}=0.000)$, differentiation grade $(r=0.533, P=0.000)$, tumor residues after primary surgery $(r=0.202, P=0.032)$, and chemotherapy sensitivity $(\mathrm{r}=0.284, \mathrm{P}=0.003)$ were significantly correlated with MSI-1 expression were positively correlated with MSI-1 expression (Table 1).

The high expression of MSI-1 is significantly correlated with poorer prognostic indicator such as higher serum CA125 levels, advanced FIGO stage, lower tumor differentiation, suboptimal primary surgery and poorer chemotherapy sensitivity, suggesting that increased MSI-1expression predicts a poorer prognosis of ovarian cancer patients and there may be a link between MSI-1 expression and paclitaxel resistant in ovarian cancer cells.

\section{MSI- 1 interference by siRNA}

When A2780 and A2780/Taxol cells were transfected with S1, S2, S3, the expressions of MSI-1 mRNA was decreased, with S2 yielding the most effective interference effect (Figure 3A,3C). Compared to the negative control group, MSI- 1 mRNA was decreased with S2 transfection by $96.84 \%$ and $95.18 \%$ in A2780 and A2780/Taxol cells, respectively. Similarly, the levels of MSI-1 protein in A2780 cells and A2780/Taxol cells decreased significantly after S2 transfection.There was also a significant difference compared to control with S1 and S3 transfection (Figure 3B,3D), but the results showed that S2 yielded the best MSI-1 knockdown effect.

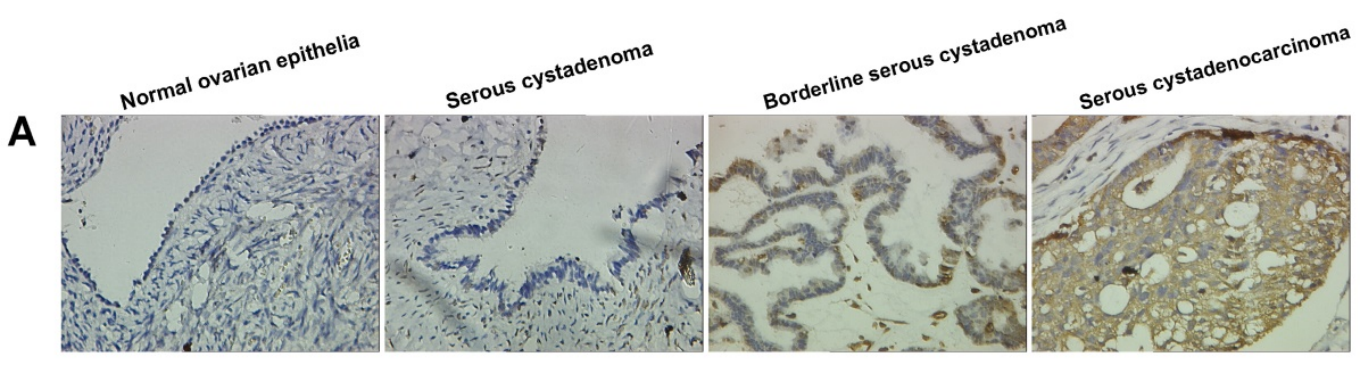

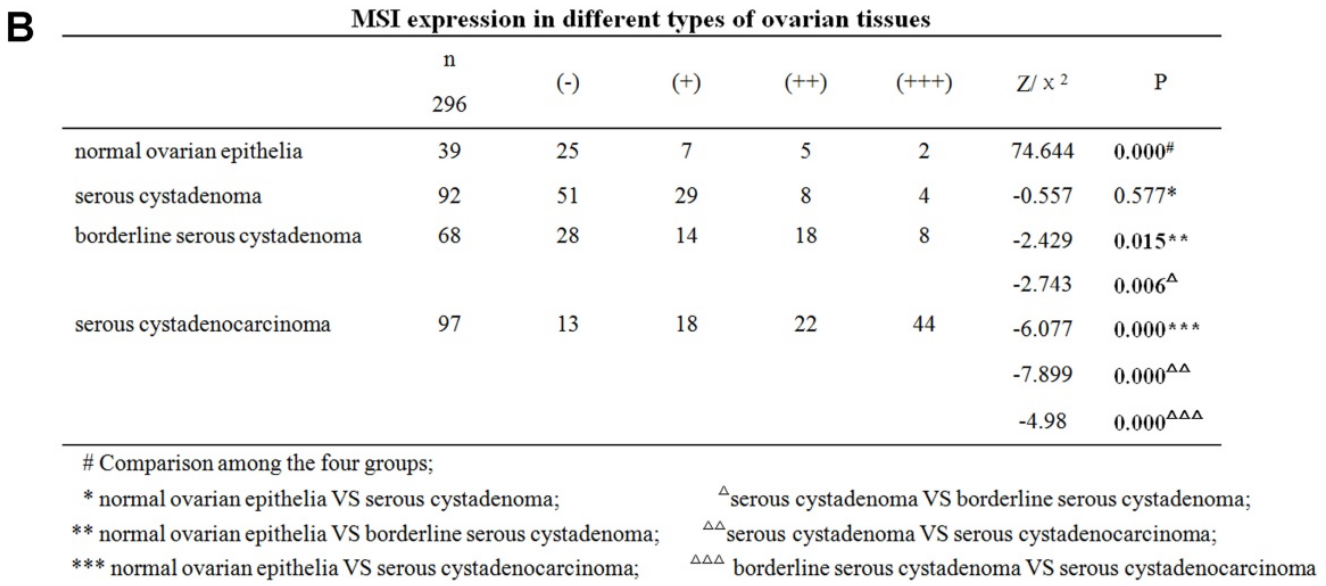

Figure 2. Increased expression of MSI-1 in serous cystadenocarcinoma. (A) MSI-1 expression and localization in normal ovarian epithelia, serous cystadenoma, borderline serous cystadenoma, and serous cystadenocarcinoma. (B) MSI expression in different ovarian tissues. 
A

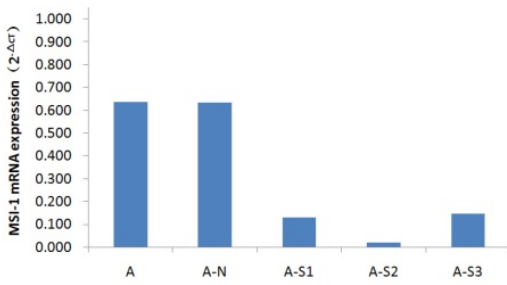

C

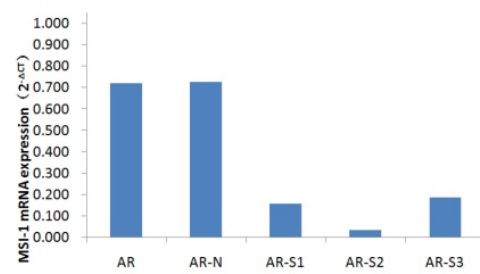

E

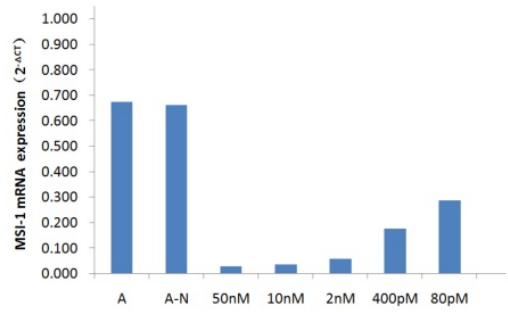

G

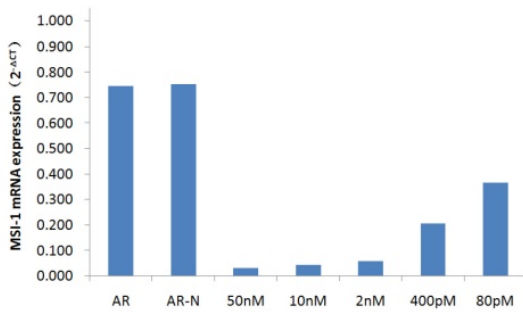

B

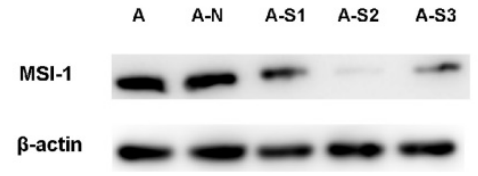

D
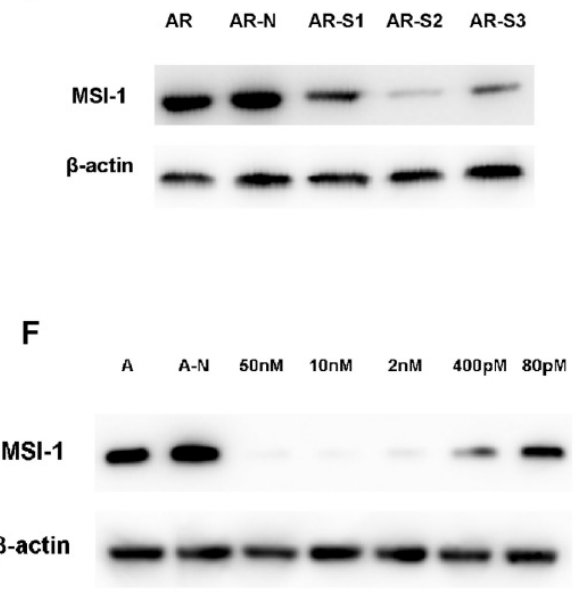

H

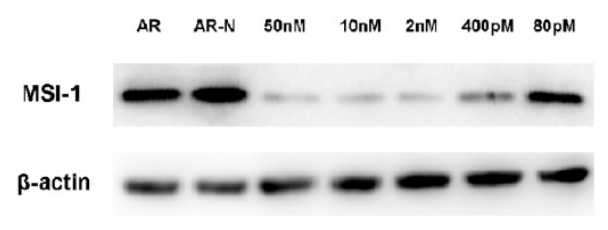

Figure 3. siRNA interference effect screening and dose optimization. (A) MSI-1mRNA expression in A2780 cells when transfected with S1, S2, S3 for $48 \mathrm{~h}$ (qPCR). A: no siRNA (Blank control); A-N: non-specific control siRNA (Negative control); A-S1: 50nM S1; A-S2: 50nM S2; A-S3: 50nM S3. (B) MSI-1 protein expression in A2780 cells when transfected with S1, S2, S3 for 72h (WB). A: no siRNA (Blank control); A-N: non-specific control siRNA (Negative control); A-S1: 50nM S1; A-S2; 50nM S2; A-S3: 50nM S3. (C) MSI-1mRNA expression in A2780/Taxol cells when transfected with S1, S2, S3 for 48h (qPCR). AR: no siRNA (Blank control); AR-N: non-specific control siRNA (Negative control); AR-S1: 50nM S1; AR-S2: 50nM S2; AR-S3: 50nM S3. (D) MSI-1 protein expression in A2780/Taxol cells when transfected with S1, S2, S3 for 72h (WB). AR: no siRNA (Blank control); AR-N: non-specific control siRNA (Negative control); AR-S1: 50nM S1; AR-S2: 50nM S2; AR-S3: 50nM S3. (E) MSI-1 mRNA expression in A2780 cells when transfected with gradually decreasing dose of S2. A: no siRNA (Blank control); A-N: non-specific control siRNA (Negative control). (F) MSI-1 protein expression in A2780 cells when transfected with gradually decreasing dose of S2. A: no siRNA (Blank control); A-N: non-specific control siRNA (Negative control). (G) MSI-1mRNA expression in A2780/Taxol cells when transfected with gradually decreasing dose of S2. A: no siRNA (Blank control); AR-N: non-specific control siRNA (Negative control). (H) MSI-1 protein expression in A2780/Taxol cells when transfected with gradually decreasing dose of S2. AR: no siRNA (Blank control); AR-N: non-specific control siRNA (Negative control).

It is known that the smaller effective dose of siRNA provides the higher specificity and the less toxic side effects. Therefore, we selected five doses (50 $\mathrm{nM}, 10 \mathrm{nM}, 2 \mathrm{nM}, 400 \mathrm{pM}$, and $80 \mathrm{pM}$ ) to determine the minimum dose.

With the gradient doses of S2 $(50 \mathrm{nM}, 10 \mathrm{nM}, 2$ $\mathrm{nM}, 400 \mathrm{pM}, 80 \mathrm{pM})$, MSI-1 mRNA was decreased by $95.78 \%, 94.72 \%, 91.25 \%, 73.45 \%$ and $56.71 \%$, respectively, in A2780 cells, and decreased by $95.74 \%, 94.15 \%$, $92.29 \%, 72.61 \%$ and $51.20 \%$, respectively in A2780/ Taxol cells (Figure 3E,3G). S2 can inhibit more than $90 \%$ of MSI- 1 mRNA expression at low concentrations $(2 \mathrm{nM})$. Even at the lowest concentration $(80 \mathrm{pM})$, S2 was still able to achieve the satisfactory inhibitory effect of nearly $50 \%$. Similarly, when the concentration of S2 was 2nM, the levels of MSI protein was decreased $91.23 \%$ and $91.03 \%$ in A2780 cells and A2780/Taxol cells, respectively. Even at $80 \mathrm{pM}$, S2 decreased MSI-1 protein levels $58.91 \%$ and $50.66 \%$ in A2780 and A2780/Taxol cells, respectively (Figure $3 \mathrm{~F}, 3 \mathrm{H})$.

\section{Proliferation, apoptosis, migration and invasion of cancer cells with MSI-1 knockdown}

The expression of MSI-1 was weak in Mrc-5 and ECV304, and was moderate in ovarian cancer cell 
3Ao. MSI-1 expression was strongly positive in endometrial cancer cell IK. We found that pLKO.1MSI-1-S2 plasmid tranfection can effectively interfere with the expression of MSI-1 protein in various cell lines. (Figure 4A-4F).

Proliferation assay showed that after transfection with pLKO.1-MSI-1-S2, the number of 3Ao, IK, A2780 and A2780/Taxol cells was significantly reduced $55.10 \%, 66.67 \%, 72.60 \%$ and $65.02 \%$, respectively. Meanwhile, suspended cells and cell debris of those tumor cells were increased under the microscope. However, we also found that for normal cell lines, such as lung fibroblasts cell Mrc-5 and umbilical vein endothelial cell ECV304, interference with MSI-1 expression had little effect on cell proliferation, which was $20.12 \%$ and $10.78 \%$, respectively, and the cell morphology did not change significantly. (Figure 5A, 5B).

We next measured apoptosis of A2780 and A2780/Taxol cells after transfection with pLKO.1MSI-1-S2 (Figure 5C). Caspase 3 activity of A2780 and A2780/Taxol cells was increased 2.29 and 2.33 fold,
A

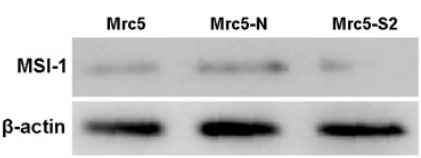

B

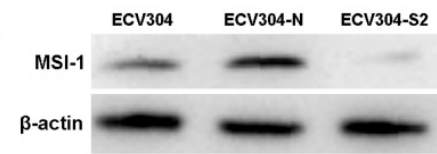

C

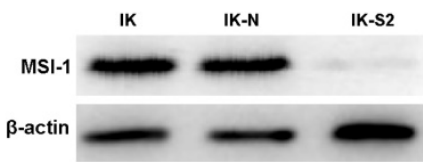

D

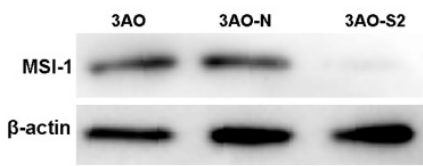

E

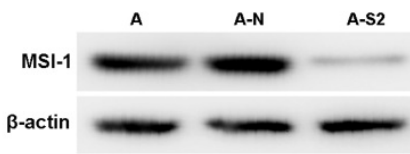

$\mathbf{F}$

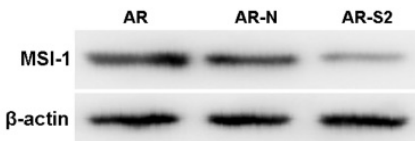

G

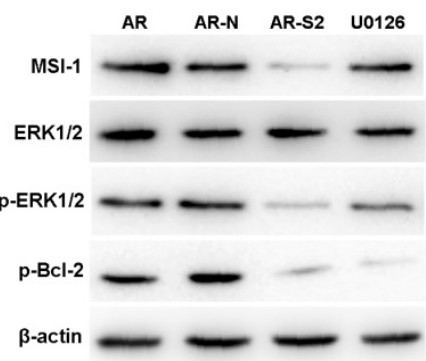

Figure 4. ERK signaling is involved in MSI-1-induced activation of antiapoptotic protein Bcl-2 and responsible for the acquirement of paclitaxel resistance in A2780/Taxol cells. (A) MSI-1 protein expression in Mrc-5 cells when transfected with pLKO.1-MSI-1- S2 (WB). Mrc5: no plasmid (Blank control); Mrc5-N: pLKO.1 puro plasmid (Negative control); Mrc5-S2: pLKO.1-MSI-1- S2. (B) MSI-1 protein expression in ECV304 cells when transfected with pLKO.1-MSI-1- S2 (WB). ECV304: no plasmid; ECV304-N: pLKO.1 puro plasmid; ECV304-S2: pLKO.1-MSI-1-S2. (C) MSI-1 protein expression in IK cells when transfected with pLKO.1-MSI-1- S2 (WB). IK: no plasmid; IK-N: pLKO.1 puro plasmid; IK -S2: pLKO.1-MSI-1S2. (D) MSI-1 protein expression in 3Ao cells when transfected with pLKO.1-MSI-1- S2 (WB). 3AO: no plasmid; 3AO-N: pLKO.1 puro plasmid; 3AO -S2: pLKO.1-MSI-1- S2. (E) MSI-1 protein expression in A2780 cells when transfected with pLKO.1-MSI-1- S2 (WB). A: no plasmid; A-N: pLKO.1 puro plasmid; A-S2: pLKO.1-MSI-1- S2. (F) MSI-1 protein expression in A2780/Taxol cells when transfected with pLKO.1-MSI-1-S2 (WB). AR: no plasmid; AR-N: pLKO.1 puro plasmid; AR-S2: pLKO.1-MSI-1- S2. (G) MSI-1 depletion decreased the activation of $\mathrm{pBCl}-2$ through ERK signal pathway (WB). AR: A2780/Taxol cells tansfected with no plasmid (Blank control); AR-N: A2780/Taxol cells tansfected with pLKO.1 puro plasmid (Negative control); AR-S2: A2780 cells tansfected with pLKO.1-MSI-1- S2; U0126: A2780/Taxol cells were treated with U0126 (10uM) for $15 \mathrm{~min}$.
In addition, change of migratory ability with MSI-1 knockdown was measured with a woundhealing assay. A2780 and A2780/Taxol cells with MSI-1 knockdown from transfection with pLKO.1MSI-1-S2 migrated and filled the wound more slowly than cells transfected with empty vector control (Figure 6A,6B). Furthermore, a cell migration assay using transwell inserts showed that the number of A2780 and A2780/Taxol cells able to penetrate the chamber was decreased $(81 \pm 11$ and $69 \pm 12$, respectiveely) compared to the control group $(272 \pm 25$ and

MSI-1 knockdown with pLKO.1-MSI-1-S2 transfection also decreased cell invasion, where the ber of A2780 and A2780/Taxol cells able to group (178 \pm 19 and $162 \pm 21$, respectively) (Figure 6D).

\section{Inhibition of MSI-1 expression reverses} paclitaxel resistance in ovarian carcinoma cells

The IC50 of A2780/Taxol cells $(38.95 \pm 3.87 \mu \mathrm{g} / \mathrm{ml}) \quad$ was significantly higher than that of the parental A2780 cells $(1.302 \pm 0.24 \mu \mathrm{g} / \mathrm{ml}$ ) (Figure 5D). In addition, the RF was 29.92, indicating that the sensitivity of A2780/Taxol cells to paclitaxel was significantly lower than that of the parental A2780 cells. When A2780/Taxol cells were transfected with pLKO.1-MSI-1-S2, the sensitivity to paclitaxel was markedly increased (IC50 $=4.32$ $\pm 0.96 \mu \mathrm{g} / \mathrm{ml})$, and the RF and RI were 3.32 and 9.08, respectively. Therefore, resistance of A2780/Taxol to paclitaxel is dramatically reversed when MSI-1 expression is inhibited.

\section{ERK signaling is involved in the MSI-1-induced activation of $\mathrm{Bcl}-2$}

When MSI-1 expression was knocked down with pLKO.1-MSI-1-S2 transfection in A2780/Taxol cells, although the overall levels of ERK did not change significantly, phosphorylation of ERK (p-ERK) was markedly inhibited (Figure $4 \mathrm{G})$. In addition, phosphorylation of the downstream gene, Bcl-2, was also decreased. To further confirm whether inhibition of ERK activation could decrease the phosphorylation of Bcl-2, A2780/Taxol cells were treated with the highly specific MEK1/2 inhibitor U0126. We found that U0126 effectively reduced p-ERK and 
p-Bcl-2 levels, and Caspase 3 activity was increased. These data shows that MSI-1 inhibits paclitaxel-induced apoptosis by enhancing the activation of Bcl-2 through activation of the ERK signaling pathway.

\section{MSI-1 Knockdown Inhibits Tumor Growth in Xenografts in SCID mice}

To explore whether MSI-1 can affect tumor growth and paclitacel-resistance in vivo, we inoculated A2780/Taxol cells as drug-resistant xenografts with or without MSI-1-S2 knockdown. The tumor volume of dominant masses was measured after SCID mice were sacrificed. The mean tumor volumes in SCID mice receiving MSI-1-S2 was $147.9 \mathrm{~mm}^{3}$, significantly smaller $(91.8 \%)$ than those in the negative control group (1813.2 $\mathrm{mm}^{3}$ ) (Figure 7). These findings further support our hypothesis that MSI-1 may play a functional role in the malignant transformation and paclitaxel-resistance of ovarian cancer.

\section{Discussion}

$\mathrm{OC}$ is characterized by few early symptoms and a poor prognosis [22]. Due to the lack of specific early symptoms, more than $60 \%$ of women diagnosed with OC are advanced stage (clinical stage III or stage IV).Even if a patient with advanced stage is treated with a combination of operation and chemotherapy, the treatment effect of therapy is debatable. One of the most important causes of poor tumor treatment and death in patients with ovarian cancer is the prevalence of chemoresistance, especially in relapsed patients. Paclitaxel is widely used as a microtubule inhibitor for the chemotherapy of ovarian cancer and other solid tumors. Many patients with OC who initially respond well to chemotherapy eventually relapse due to chemoresistance [23]. Research on the key molecules and mechanisms involving in drug sensitivity is very important and necessary to reverse chemoresistance in OC therapy.
A
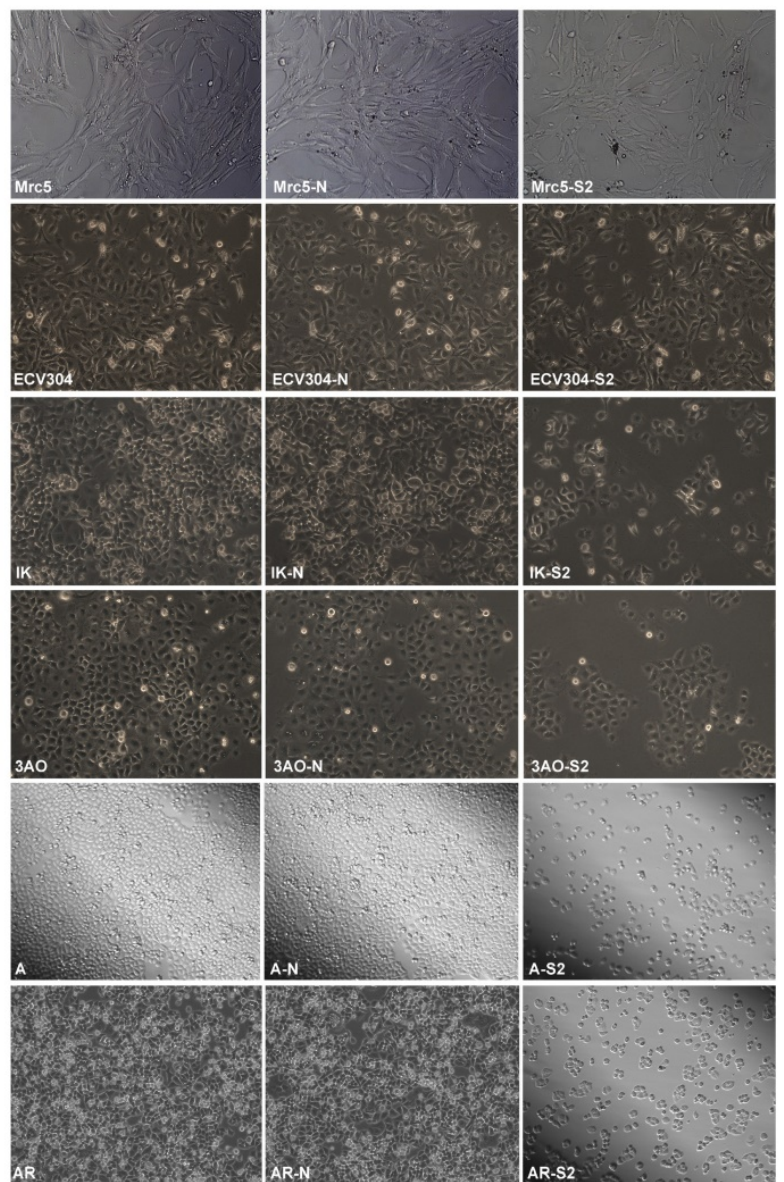

Figure 5. MSI-1 knockdown increased the apoptosis and recoveried paclitaxel sensitivity in A2780/Taxol cells. (A) Cell proliferation and the morphologic characteristic under an inverted microscope. (B) Cell proliferation Assay (colorimetric, BrdU). (C) Caspase-3 activity assay. (D) The sensitivity to paclitaxel was restored by transfection with pLKO.1-MSI-1- S2. Mrc5: no plasmid, Mrc5-N: pLKO.1 puro plasmid, Mrc5-S2: pLKO.1-MSI-1- S2; ECV304: no plasmid, ECV304-N: pLKO.1 puro plasmid, ECV304-S2: pLKO.1-MSI-1- S2; IK: no plasmid, IK-N: pLKO.1 puro plasmid, IK -S2: pLKO.1-MSI-1- S2; 3AO: no plasmid, 3AO-N: pLKO.1 puro plasmid, 3AO -S2: pLKO.1-MSI-1-S2; A: no plasmid, A-N: pLKO.1 puro plasmid, A-S2: pLKO.1-MSI-1-S2; AR: no plasmid, AR-N: pLKO.1 puro plasmid, AR-S2: PLKO.1-MSI-1-S2. 
A

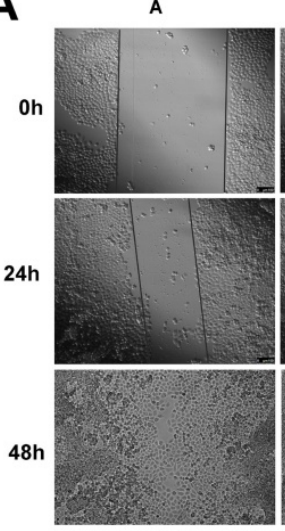

C

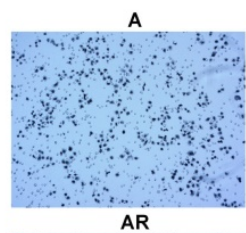

AR

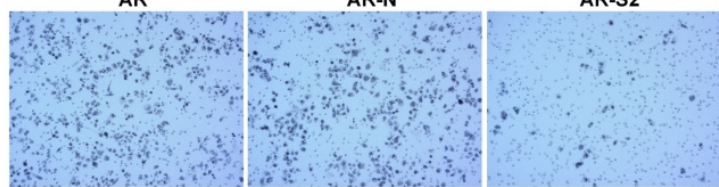

B

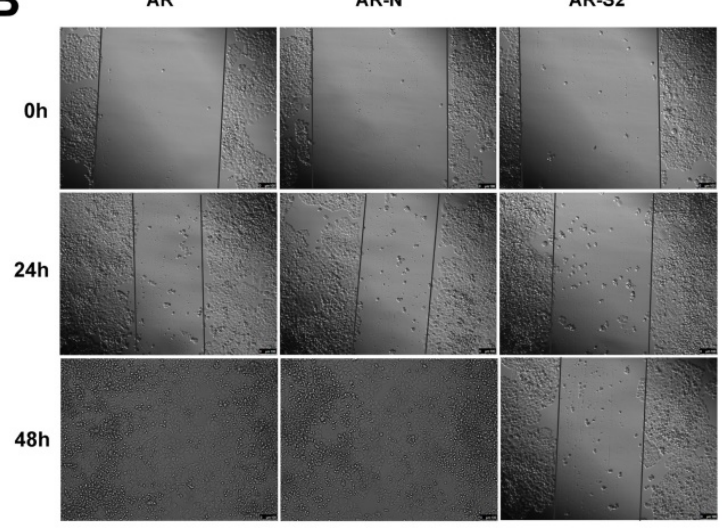

D
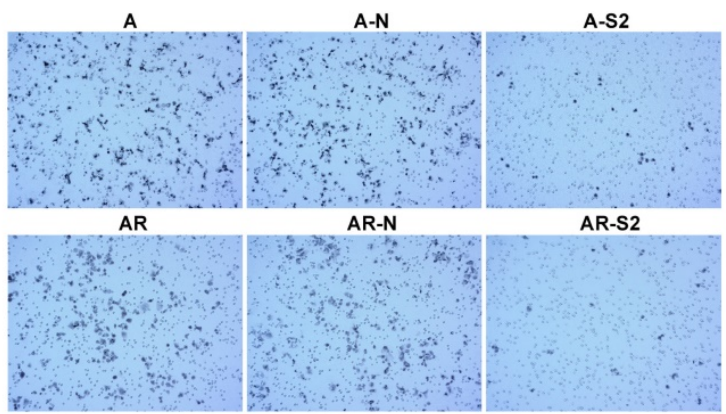

Figure 6. Migration and invasion of cancer cells after blocking the expression of MSI-1. (A) Wound-healing assay in A2780 cells. (B) Wound-healing assay in A2780/Taxol cells. (C) Migration assay. (D) Matrigel invasion assay. A: A2780 cells tansfected with no plasmid (Blank control); A-N: A2780 cells tansfected with pLKO.1 puro plasmid (Negative control); A-S2: A2780 cells tansfected with pLKO.1-MSI-1- S2; AR: A2780/Taxol cells tansfected with no plasmid (Blank control); AR-N: A2780/Taxol cells tansfected with pLKO.1 puro plasmid (Negative control); AR-S2: A2780 cells tansfected with pLKO.1-MSI-1- S2

Stem cells can differentiate into other type tissue cell. Similarly, there are different degree cancer cells in the same tissue. Usually, the better the differentiation of cancer cells is, the lower the malignancy is. There are many similarities between the tumor cells and the stem cells. Cancer stem cells may originate from transformed normal stem cells. Therefore, identifying cancer stem cell-related molecules can not only identify novel markers for early diagnosis, but can also play an important role in ovarian cancer drug-resistance. These cells maintain their undifferentiated state through the regulation of several downstream molecules to provide a limitless potential of self-renewal, invasion and metastasis [24,25]. Therefore, efforts into research on multi-potential and differentiation-related molecules maybe provide early prognostic and diagnostic strategy in ovarian cancer.

MSI-1 is regarded as a stem cell marker and the high expression of MSI-1 in multiple solid tumor cell lines suggests that the protein may have a broader role in solid tumors [26]. Studies show that highly enriched MSI-1 is often found in cancer cells, such as in malignant gliomas, astrocytomas, colorectal cancer and endometrial carcinoma $[1,12,27,28]$. Importantly, in some cancer types such as gliomas and leukaemia, increased expression of MSI-1 has been found to correlate with tumor progression and ultimately to a poor prognosis [29-31]. In our study, to investigate the role of MSI-1 in the development of ovarian cancer and chemoresistance, we examined expression of MSI-I protein in 39 normal ovarian epithelial tissues, 92 serous cystic adenomas, 68 borderline serous cystadenomas, and 97 serous cystadenocarcinomas by immunohistochemistry. The expression of MSI-1 in serous cystadenocarcinomas was found significantly increased, especially in chemoresistant patients compared with chemosensitive ones. In addition, clinical pathological correlation analysis showed that high expression of MSI-1was associated with poor prognostic factors, including higher serum CA125 levels, advanced FIGO stage, lower tumor differentiation, and suboptimal primary surgery and chemotherapy resistance. To the best of our knowledge, exploring the role of MSI-1 in paclitaxel resistance of ovarian cancer, we are the first report. Although MSI-1 has been studied in many tumors, including glioma, colorectal cancer, endometrial carcinoma, and so on [10-12], the expression or the role of MSI-1 in ovarian cancer has not been reported so far. In addition, although MSI-1 has some research on tumor chemoresistance, for example, studies have found that 
MSI-1 inhibits drug-induced apoptosis through AKT/IL6 regulatory circuit, thus plays a regulatory role in tumor cell proinflammatory circuits formation, chemoresistance, and tumor recurrence [13], it is not known whether MSI-1 has a role in paclitaxel chemoresistance. Paclitaxel is currently the most effective chemotherapy for solid tumors and the most widely used anti-microtubule chemotherapy drugs. It is used in ovarian cancer, breast cancer, lung cancer, head and neck cancer, esophageal cancer, gastric cancer and other soft tissue sarcoma. It is considered to be one of the most effective anticancer drugs for humans in the future. Therefore, studying the role of MSI-1 in paclitaxel resistance and finding a solution to paclitaxel resistance is of great significance.

Given the demonstrated important role of MSI-1 in the pathogenesis of ovarian carcinoma, we aimed to find an effective method to suppress MSI-1 protein expression, and therefore used the siRNA interfernce to achieve this goal. We designed three pairs of siRNAs against the MSI-1 gene, and the most effect pair of siRNAs was chosen for further optimization of

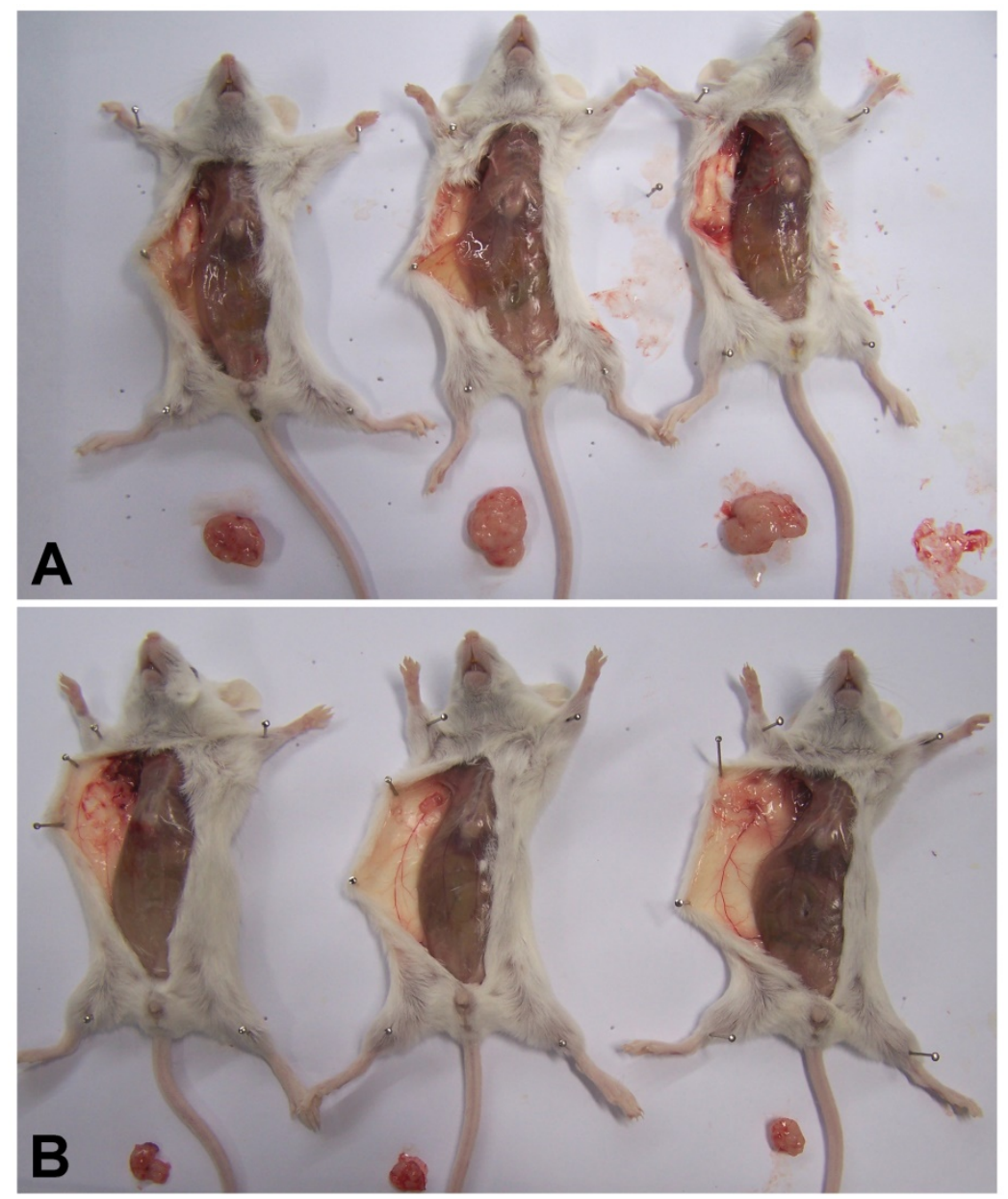

Figure 7. MSI-1 knockdown inhibits Paclitaxel-resistant xenograft tumor growth in vivo. (A) Control group: treated with negative control siRNA $(1 \mathrm{mg} / \mathrm{kg})$ every 3 days for 5 weeks; (B) Treatment group: treated with MSI-1-siRNA2 $(1 \mathrm{mg} / \mathrm{kg})$ every 3 days for 5 weeks. silencing and determining the ideal interference siRNA concentration. Research has shown that the lowest concentration of siRNA that can achieve knockdown gives higher specificity and less toxic side effects. Our results showed that even at low concentrations $(2 \mathrm{nM})$, the siRNA selected against MSI-1 inhibited more than 90\% of MSI-1 expression.

We evaluated the role of MSI-1 in proliferation, apoptosis, migration and invasion of OC cells. The knockdown of MSI-1 protein expression led to decreased proliferation, increased apoptosis, and reduced migration and invasion. Moreover, MSI-1 knockdown rescued the drug sensitivity to paclitaxel $(\mathrm{RF}=3.32, \mathrm{RI}=9.08)$, theses result indicated that MSI-1 may play an important role in paclitaxel resistance in OC cells.

Although a positive correlation between MSI-1 expression in OC tissues and clinical progression was observed, and MSI-1 was observed to play an important role in paclitaxel resistance and altered cancer cell behavior, we questioned why the expression of MSI-1 was not significantly different between paclitaxel-

resistant cells and paclitaxel-sensitive parent cells. We speculate that the high expression of MSI-1 is because of congenital inheritance [32].One reason that A2780 cells possessing paclitaxelresistant is due to the abnormally high expression of MSI-1 in this cells.

Studies have shown that MSI-1 overexpression is correlated with the disruption of signaling pathways involved in processes such as cell proliferation and cell fate decisions [11,33-35]. It is also reported that MSI-1 modulates mammary progenitor cell expansion through its ability to coordinate cell cycle entry and activate the Notch and Wnt pathways through a novel autocrine process involving PLF1 and DKK3 [27]. In our study, the expression of p-ERK and p-Bcl2, which are both involved in anti-apoptotic signaling, were decreased with MSI-1 knockdown and with treatment with a highly specific MEK1/2 inhibitor (U0126). These suggest that ERK signaling is involved in MSI-1-mediated paclitaxel resistance in ovarian carcinoma cells. MSI-1 inhibits paclitaxel-induced apoptosis by activating ERK and Bcl2, and inhibition of MSI-1 can reverse the paclitaxel-resistant cells to sensitive paclitaxel again. 
To confirm the effects of MSI-1 which were found in vitro functional studies, we established a mouse subcutaneous tumor drug resistance model treated with or without MSI-1 knockdown. Suppression of MSI-1 by siRNA resulted in a significant inhibition of tumor volume increase, and the tumor growth was inhibited.

In summary, MSI-1 is highly expressed in ovarian carcinoma cells, and high MSI-1 expression is associated with poor prognosis of OC patients. Knockdown of MSI-1 inhibits proliferation, promotes apoptosis, and reduces migration and invasion of cancer cells. Furthermore, blocking the expression of MSI-1 can reduce the expression of $\mathrm{p}$-Bcl2 by downregulating $\mathrm{p}$-ERK, and can consequently promote paclitaxel sensitivity in ovarian cancer cells. Our findings suggest that MSI-1 may act as a promoter of chemoresistance as well as a paclitaxel response marker, and possible to be used as a new therapeutic target for reversing paclitaxel resistance in recurrent case of ovarian carcinoma.

\section{Abbreviations}

OC: ovarian cancer; PROC: paclitaxel resistance ovarian cancer; siRNA: small interfering RNA; MSI-1: Musashi-1; CSCs: cancer stem cells; Taxol: Trade name of paclitacel; pLKO.1-MSI-1-S2: pLKO.1-puro inserted with MSI-1-S2 sequence; qPCR: real-time quantitative PCR; RF: resistant folder; RI: reversal index.

\section{Supplementary Material}

Supplementary tables.

http://www.jcancer.org/v10p1580s1.pdf

\section{Acknowledgement}

We thank Minghua Yu for friendly support with the immunohistochemical experiment, thank Junyan Ma for friendly support with the qPCR experiment.

\section{Funding}

This work was supported by grants from the National Nature Science foundation of China (No.309 73380) and Zhejiang Provincial Natural Science Foundation of China (No. Y2110200).

\section{Authors' contributions}

Conceptualization: Feng Ye, Huaizeng Chen, Jia Liu, Hanzhi Wang, Qi Cheng, Caiyun Zhou, Xiaojing Chen. Data curation: Feng Ye. Formal analysis: Huaizeng Chen, Feng Ye. Funding acquisition: Huaizeng Chen, Feng Ye. Methodology: Huaizeng Chen, Feng Ye, Jia Liu, Hanzhi Wang, Qi Cheng, Caiyun Zhou, Xiaojing Chen. Supervision: Qi Cheng, Caiyun Zhou. Writing original draft: Feng Ye. Writing and review \& editing: Huaizeng Chen, Feng Ye, Jia Liu, Hanzhi Wang,Qi Cheng, Caiyun Zhou, Xiaojing Chen.

\section{Competing Interests}

The authors have declared that no competing interest exists.

\section{References}

1. Siegel R, Naishadham D, Jemal A. Cancer statistics, 2012. CA Cancer J Clin. 2012;62(1):10-29.

2. Cho KR, Shih IeM. Ovarian cancer. Annu Rev Pathol. 2009;4:287-313.

3. Kurman RJ, Shih IeM. Molecular pathogenesis and extraovarian origin of epithelial ovarian cancer--shifting the paradigm. Hum Pathol. 2011;42(7):918-31.

4. He QZ, Luo XZ, Wang K, et al. Isolation and characterization of cancer stem cells from high-grade serous ovarian carcinomas. Cell Physiol Biochem. 2014;33(1):173-84

5. Vathipadiekal V, Saxena D, Mok SC,et al. Identification of a potential ovarian cancer stem cell gene expression profile from advanced stage papillary serous ovarian cancer. PLoS One. 2012;7:e29079.

6. Hemmati HD, Nakano I, Lazareff JA, et al. Cancerous stem cells can arise from pediatric brain tumors. Proc Natl Acad Sci USA. 2003;100(25):15178-15183.

7. Okabe M, Imai T, Kurusu M, et al. Translational repression determines a neuronal potential in Drosophila asymmetric cell division. Nature. 20014;11(6833):94-98.

8. Nakamura M, Okano H, Blendy JA,et al. Musashi, a neural RNA-binding protein required for Drosophila adult external sensory organ development. Neuron. 1994;13(1): 67-81

9. Imai $\mathrm{T}$, Tokunaga $\mathrm{A}$, Yoshida $\mathrm{T}$, et al. The neural RNA-binding protein Musashi1 translationally regulates mammalian numb gene expression by interacting with its mRNA. Mol Cell Biol. 2001;21(12):3888-3900.

10. Götte M, Wolf M, Staebler A, et al. Increased expression of the adult stem cell marker Musashi-1 in endometriosis and endometrial carcinoma. J Pathol. 2008;215(3):317-329.

11. Götte M, Greve B, Kelsch R, et al. The adult stem cell marker musashi-1 modulates endometrial carcinoma cell cycle progression and apoptosis via notch-1 and p21(WAF1/CIP1). Int J Cancer. 2011;129(8):2042-2049.

12. Okano $\mathrm{H}$, Imai $\mathrm{T}$, Okabe M. Musashi: a translational regulator of cell fate. J Cell Sci. 2002; 115(Pt 7): 1355-1359.

13. Chen HY, Lin LT, Wang ML, et al. Musashi-1regulates AKT-derived IL-6 autocrinal/paracrinal malignancy and chemoresistancein glioblastoma. Oncotarget. 2016;7(27):42485-42501.

14. Han X, Du F, Jiang L, et al. A2780 human ovarian cancer cells with acquired paclitaxel resistance display cancer stem cell properties. Oncol Lett. 2013;6(5):1295-1298

15. Bapat SA, Mali AM, Koppikar CB, et al. Stem and progenitor-like cells contribute to the aggressive behavior of human epithelial ovarian cancer. Cancer Res. 2005;65(8): 3025-3029.

16. Zhang J, Li YL, Zhou CY, et al. Expression of octamer-4 in serous and mucinous ovarian carcinoma. J Clin Pathol. 2010;63(10):879-883.

17. Pan Y, Jiao J, Zhou C, et al. Nanog is highly expressed in ovarian serous cystadenocarcinoma and correlated with clinical stage and pathological grade. Pathobiology. 2010;77(6):283-288.

18. Ye F, Li Y, Hu Y, et al. Stage-specific embryonic antigen 4 expression in epithelial ovarian carcinoma. Int J Gynecol Cancer. 2010;20(6):958-964.

19. Ye F, Li Y, Hu Y, et al. Expression of Sox2 in human ovarian epithelial carcinoma. J Cancer Res Clin Oncol. 2011;137(1):131-137.

20. Ye F, Li Y, Hu Y, et al. Expression of Sox2 in human ovarian epithelial carcinoma. J Cancer Res Clin Oncol. 2011;137(1):131-137.

21. Tomayko MM, Reynolds CP. Determination of subcutaneous tumor size in athymic (nude) mice. Cancer Chemother Pharmacol. 1989;24(3):148-54.

22. Hartge P. Designing early detection programs for ovarian cancer. J Natl Cancer Inst. 2010;102(1):3-4.

23. Hassan MK, Watari H, Christenson L, et al. Intracellular clusterin negatively regulates ovarian chemoresistance: compromised expression sensitizes ovarian cancer cells to paclitaxel. Tumour Biol. 2011;32(5):1031-1047.

24. Boyer LA, Lee TI, Cole MF, et al. Core transcriptional regulatory circuitry in human embryonic stem cells. Cell. 2005;122(6):947-956.

25. Wang J, Rao S, Chu J, et al. A protein interaction network for pluripotency of embryonic stem cells. Nature. 2006; 444(7117):364-368.

26. Shu HJ, Saito $\mathrm{T}$, Watanabe $\mathrm{H}$, et al. Expression of the Musashi1 gene encoding the RNA-binding protein in human hepatoma cell lines. Biochem Biophys Res Commun. 2002;293(1):150-154

27. Wang XY, Yin Y, Yuan H,et al. Musashi1 modulates mammary progenitor cell expansion through proliferin-mediated activation of the Wnt and Notch pathways. Mol Cell Biol. 2008;28(11):3589-3599.

28. Wang XY, Penalva LO, Yuan H, et al. Musashi1 regulates breast tumor cell proliferation and is a prognostic indicator of poor survival. Mol Cancer. 2010;9:221. 
29. Ito T, Kwon HY, Zimdahl B, et al. Regulation of myeloid leukaemia by the cell-fate determinant Musashi. Nature. 2010;466(7307):765-768.

30. Kharas MG, Lengner CJ, Al-Shahrour F, et al. Musashi-2 regulates normal hematopoiesis and promotes aggressive myeloid leukemia. Nat Med. 2010;16(8):903-908.

31. Griner LN, Reuther GW. Aggressive myeloid leukemia formation is directed by the Musashi 2/Numb pathway. Cancer Biol Ther. 2010;10(10): 979-982.

32. Hino O, Kobayashi T. Mourning Dr. Alfred G. Knudson: the two-hit hypothesis, tumor suppressor genes, and the tuberous sclerosis complex. Cancer Sci. 2017;108(1):5-11.

33. Rezza A, Skah S, Roche C, et al. The overexpression of the putative gut stem cell marker Musashi-1 induces tumorigenesis through Wnt and Notch activation. J Cell Sci. 2010;123(Pt 19):3256-3265.

34. Spears E, Neufeld KL. A novel double-negative feedback loop between adenomatous polyposis coli and musashi1 in colon epithelia. J Biol Chem. 2011;286(7) :4946-4950.

35. Muto J, Imai T, Ogawa D, et al. RNA-binding protein Musashi1 modulates glioma cell growth through the post-transcriptional regulation of Notch and PI3 kinase/Akt signaling pathways. PLoS One. 2012;7(3):e33431 